\title{
Alanyl-glutamine protects the intestinal barrier function in trained rats against the impact of acute exhaustive exercise
}

\author{
A.K.L. Freitas ${ }^{1}{ }^{1}$, M.T.B. Silva $\oplus^{2}{ }^{2}$, C.M.S. Silva ${ }^{1}{ }^{1}$, M.M.G. Prata ${ }^{1}{ }^{1}$, F.A.P. Rodrigues $\oplus^{3}{ }^{3}$,

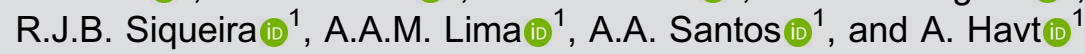 \\ ${ }^{1}$ Departamento de Fisiologia e Farmacologia, Faculdade de Medicina, Universidade Federal do Ceará, \\ Fortaleza, CE, Brasil \\ ${ }^{2}$ Departamento de Educação Física, Universidade Federal do Piauí, Teresina, PI, Brasil \\ ${ }^{3}$ Departamento de Educação Física e Esporte, Instituto Federal de Educação, Ciência e Tecnologia do Ceará, \\ Fortaleza, CE, Brasil
}

\begin{abstract}
Strenuous exercise triggers deleterious effects on the intestinal epithelium, but their mechanisms are still uncertain. Here, we investigated whether a prolonged training and an additional exhaustive training protocol alter intestinal permeability and the putative effect of alanyl-glutamine (AG) pretreatment in this condition. Rats were allocated into 5 different groups: 1) sedentary; 2 and 3 ) trained (50 min per day, 5 days per week for 12 weeks) with or without 6 weeks oral (1.5 g/kg) AG supplementation; 4 and 5) trained and subjected to an additional exhaustive test protocol with or without oral AG supplementation. Venous blood samples were collected to determine gasometrical indices at the end of the 12-week protocol or after exhaustive test. Lactate and glucose levels were determined before, during, and after the exhaustive test. lleum tissue collected after all experimental procedures was used for gene expression analysis of Zonula occludens 1 (ZO-1), occludin, claudin-2, and oligopeptide transporter 1 (PepT-1). Intestinal permeability was assessed by urinary lactulose/mannitol test collected after the 12-week protocol or the exhaustive test. The exhaustive test decreased $\mathrm{pH}$ and base excess and increased $\mathrm{pCO}_{2}$. Training sessions delayed exhaustion time and reduced the changes in blood glucose and lactate levels. Trained rats exhibited upregulation of PEPT-1, ZO-1, and occludin mRNA, which were partially protected by AG. Exhaustive exercise induced intestinal paracellular leakage associated with the upregulation of claudin-2, a phenomenon protected by AG treatment. Thus, AG partially prevented intestinal training adaptations but also blocked paracellular leakage during exhaustive exercise involving claudin-2 and occludin gene expression.
\end{abstract}

Key words: Claudin-2; Intestinal permeability; Lactulose-mannitol test; Physical exercise; Tight junctions

\section{Introduction}

Physical activity has been prescribed for patients with gut dysmotility to improve their health (1). However, physical exercises may impair the gastrointestinal tract physiology, eliciting vomit, diarrhea, bloody stool, and gastro-esophageal reflux, especially after prolonged and strenuous activity involving heat stress (2). The impact of physical exercise on human gut motor behavior is still uncertain. There is conflicting evidence concerning its effects on gastric emptying rate (GER) (3). Such discrepancies may be explained by different experimental conditions including exercise protocols, nature of the test meal, and duration of the post-prandial interval for GER analysis. We previously reported that acute high-intensity exercise delayed the GER of a liquid test meal in awake rats, a phenomenon prevented by bicarbonate administration (4). Although the etiology of exercise-induced gastrointestinal tract dysmotility appears to be multifactorial, gut ischemia is considered the major pathophysiological mechanism (5). Strenuous physical exercise leads to blood volume redistribution, shunting blood flow away from the splanchnic area. Therefore, gut ischemia leads to tissue hypoxia, local depletion of adenosine triphosphate (ATP), acidosis, local inflammation, and formation of reactive oxygen species $(6,7)$. Physical stress is associated with a high risk of intestinal mucosal integrity loss and bacterial translocation (8). However, the precise mechanisms of increased gut permeability, in this context, are still unknown $(7,9)$. 
Glutamine is a major substrate for enterocytes and cells of the mucosal immune system and accelerates the repair of damaged intestinal mucosa and barrier function $(10,11)$. Glutamine, however, presents limited solubility and has a tendency to hydrolyze into potentially toxic glutamate. Linking glutamine to alanine has solved both drawbacks. We previously observed that alanyl-glutamine (AG) is stable, highly soluble, well tolerated, effective for sodium transport, and a key component for repairing intestinal injury both in laboratory animals and human subjects (12).

The present study evaluated whether periodized training and exhaustive exercise would alter intestinal permeability in a rat model and the putative effects of $A G$ pretreatment in the gut function. We hypothesized that $A G$ would be able to protect intestinal cells against leakage, presumably caused by strenuous exercise.

\section{Material and Methods}

\section{Animals and experimental design}

Male Wistar rats (300-350 g) were supplied by the Central Vivarium of the Federal University of Ceará (Brazil). All experimental protocols were conducted in accordance with the principles for the ethical use of animals for scientific research regulated by CONCEA (National Council for Control of Animal Experimentation), and performed after approval by the Ethical Committee for Animal Use of the Federal University of Ceará, Brazil (protocol 13/ 2013).

Rats were randomly divided into five groups ( $n=8$ each): sedentary group (S); trained group (50 min per day, 5 days per week for 12 weeks) (T); trained group (50 min per day, 5 days per week for 12 weeks) supplemented orally with AG for the last 6 weeks of swimming sessions (T-AG); trained group (50 min per day, 5 days per week for 12 weeks) subjected to an exhaustive test (ET) that took place after the 12-week swimming protocol; and trained group (50 min per day, 5 days per week for 12 weeks) supplemented orally with AG for the last 6 weeks of swimming session and subjected to an exhaustive test that took place after the 12-week swimming protocol (ET-AG). LAlanyl-glutamine (Ajinomoto ${ }^{\mathbb{R}}$, Brazil) was given by gavage $(1.5 \mathrm{~g} / \mathrm{kg})$ at the end of each daily swimming session for the last six weeks of the training protocol $(13,14)$ (Supplementary Figure S1). Animals without supplementation received only the vehicle (water) by gavage.

The physical training protocol consisted of swimming exercise in a cylindrical tank $(60 \mathrm{~cm}$ diameter, $50 \mathrm{~cm}$ depth, $30 \pm 1^{\circ} \mathrm{C}$ water temperature). All animals underwent an adaptive period. This adaptive period was done for five consecutive days, in which the animals swam freely for $10,20,30,40$, and $50 \mathrm{~min}$, respectively. After the adaptive period, rats from T, T-AG, ET, and ET-AG groups were subjected to the training period, which consisted of 50-min daily swimming sessions, from Monday to Friday for 12 weeks (15). Animals of the sedentary group were placed from Monday to Friday for 12 weeks for $10 \mathrm{~min}$ in tanks with enough water to immerse their paws $(5 \mathrm{~cm})$.

The rats of ET and ET-AG groups, exactly $24 \mathrm{~h}$ after the last session of the 12-week swimming protocol, were subjected to an additional swimming exhaustive test, without extra load $(13,16,17)$. Uncoordinated movements and a contorted body characterized the physical collapse in the exhaustive test, such that the rats could not keep their nostrils above the water for $10 \mathrm{~s}$. The extent of exhaustion time (in min) was determined for ET and ET-AG groups. Blood samples were collected from the animals' tails after performing a tiny cut of its tip, according to the technique described by Rogero et al. (13). The first sample was collected immediately before starting the exhaustive test. The second sample was collected exactly two hours after the beginning of the exhaustive test. The third one was collected right after the end of the exhaustive test. These samples served for glucose and lactate analysis performed by Accutrend Plus instrument (Roche Diagnostics, Germany).

All animals were gavage-fed with a lactulose/mannitol solution (200 and $50 \mathrm{mg} / \mathrm{mL}$, respectively) (18). The S, T, and T-AG groups received lactulose/mannitol solution after the last day of the 12-week protocol. The animals in ET and ET-AG groups received the solution after the exhaustive test. After the administration of the lactulose/ mannitol solution, all animals were individually housed in metabolic cages for $24 \mathrm{~h}$ for urine collection. Finally, all rats were euthanized under anesthesia (pentobarbital sodium $50 \mathrm{mg} / \mathrm{kg}$ ) followed by exsanguination. After death was clinically detected, ileum tissue was collected for RNA expression analysis.

\section{Blood gas analysis}

Under anesthesia, we collected a sample of venous blood from the retro-orbital plexus to measure $\mathrm{pH}$, carbon dioxide partial pressure $\left(\mathrm{pCO}_{2}\right)$, oxygen partial pressure $\left(\mathrm{pO}_{2}\right)$, base excess $(\mathrm{BE}), \mathrm{O}_{2}$ saturation $\left(\mathrm{SatO}_{2}\right)$, and bicarbonate $\left(\mathrm{HCO}_{3}{ }^{-}\right)$using a gasometer (Cobas b121, Roche Diagnostics, Germany). This procedure was executed right after the last swimming session for groups $\mathrm{S}, \mathrm{T}$, and T-AG and right after the exhaustive test for ET and ET-AG.

\section{Intestinal permeability evaluation}

Intestinal permeability was evaluated by analyzing urinary lactulose and mannitol levels (18). After returning from anesthesia for blood collection for gasometrical analysis, rats were gavage-fed with a lactulose $(200 \mathrm{mg} /$ $\mathrm{mL})$ and mannitol $(50 \mathrm{mg} / \mathrm{mL})$ solution $(1 \mathrm{~mL} / \mathrm{kg})$ and kept individually in metabolic cages for $24 \mathrm{~h}$. During this period of $24 \mathrm{~h}$, urine was collected in a flask containing $25 \mu \mathrm{L}$ of chlorhexidine solution $(40 \mathrm{mg} / \mathrm{mL})$. Total urine volume was recorded, and two aliquots were stored at $-20^{\circ} \mathrm{C}(17)$. Each urine sample $(50 \mu \mathrm{L})$ was mixed with $50 \mu \mathrm{L}$ of a melibiose solution (3.6 mM) and diluted into $2.9 \mathrm{~mL}$ of double-distilled water. After centrifugation at $562 \mathrm{~g}$ for 
10 minutes at $4^{\circ} \mathrm{C}$ and filtration, a sample was used for sugar determination by high-performance liquid chromatography (19).

\section{mRNA expression of intestinal transporters and tight junctions}

Total RNA was extracted from the ileum using the RNeasy Lipid Tissue Mini Kit (Qiagen, Germany) according to the manufacturer's instructions and then subjected to cDNA synthesis using the iScript cDNA Synthesis Kit (Bio-Rad, USA). The mRNA expression of the tight junction (Zonula occludens-1, claudin-2, and occludin) and oligopeptide transporter 1 (PepT-1) was assayed by real-time quantitative polymerase chain reaction (qPCR) using the CFX96 Touch Detection System (Bio-Rad). The tyrosine 3monooxygenase/tryptophan 5E-monooxygenase activation protein zeta polypeptide (YWHAZ) served as housekeeping gene (20). DNA primers for all the analyzed genes (Supplementary Table S1) were designed on the basis of mRNA sequences obtained from the National Centre for Biotechnology Information (http://www.ncbi.nlm.nih.gov). The data were obtained using CFX Manager 3.0 software (Bio-Rad), based on the values of the threshold cycle, in which the observed fluorescence is 10 -fold higher than the basal fluorescence for each qPCR assay. The mRNA expression was determined by applying the $2^{-\Delta \Delta C T}$ mathematical method (21).

\section{Statistical analysis}

Data are reported as means $\pm S D$. One-way analysis of variance (ANOVA) followed by Bonferroni's post hoc test was used for parametric data. mRNA expression data were evaluated by Mann-Whitney's test. $\mathrm{P}<0.05$ was considered statistically significant.

\section{Results}

\section{Parameters of exercise intensity}

Figure 1 shows the serum glucose and lactate levels of rats subjected to the exhaustive test. Serum glucose levels decreased $(P<0.05)$ in blood samples collected immediately after the exhaustive test, regardless of $A G$ supplementation. There was no difference in glucose levels between ET and ET-AG groups regardless of swimming duration during the exhaustive test. Rats of ET and ET-AG groups swam for $248.9 \pm 57.6 \mathrm{~min}$ and $246.6 \pm 52.4 \mathrm{~min}$, respectively. Animals of the ET group showed glucose levels of $107.9 \pm 5.49,107.8 \pm 6.08$, and $43.2 \pm 9.24 \mathrm{mg} / \mathrm{dL}$ before, during, and after the exhaustive test, respectively (Figure 1A). Animals from the ET-AG group showed glucose levels of $110.4 \pm 7.89,113.2 \pm 7.60$, and $38.0 \pm 5.33$ $\mathrm{mg} / \mathrm{dL}$ before, during, and after the exhaustive test, respectively (Figure 1B). Compared with their respective basal levels, serum lactate increased $(P<0.05)$ during and after the exhaustive test for both the ET and ET-AG groups. Before, during, and after the exhaustive test, the ET rats showed serum lactate levels of $2.1 \pm 0.10,3.5 \pm 0.24$, and $4.5 \pm 0.39 \mathrm{mmol} / \mathrm{L}$, respectively (Figure $1 \mathrm{C}$ ). Animals in ET-AG group presented lactate levels of $2.5 \pm 0.21 \mathrm{mmol} / \mathrm{L}$ before, $3.7 \pm 0.28 \mathrm{mmol} / \mathrm{L}$ during, and $5.1 \pm 0.55 \mathrm{mmol} / \mathrm{L}$ after the exhaustive test (Figure 1D). AG supplementation did not affect lactate metabolism (Figure 1D).
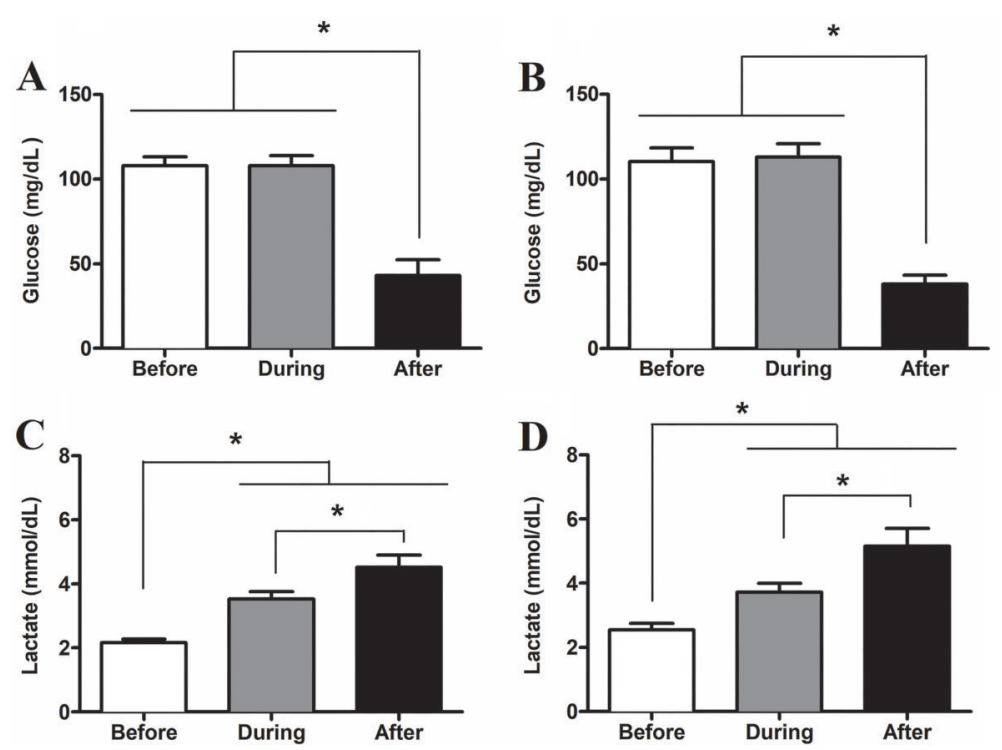

Figure 1. Serum glucose (A and $\mathbf{B}$ ) and lactate (C and $\mathbf{D})$ concentration measured before, during (50 min), and after the exhaustive test. Data for ET groups (trained animals subjected to an exhaustive test without supplementation) are shown in graphs $\mathbf{A}$ and $\mathbf{C}$. Data for ET-AG groups (trained animals subjected to exhaustive test and supplemented with alanyl-glutamine) are shown in graphs $\mathbf{B}$ and $\mathbf{D}$. The data $(n=8)$ are reported as means $\pm S D$. * $P<0.05$, ANOVA followed by Bonferroni post hoc test. 
Table 1. Gasometric analysis results.

\begin{tabular}{lrrrrr}
\hline Variables & \multicolumn{5}{c}{ Training } \\
\cline { 2 - 6 } & \multicolumn{1}{c}{$\mathrm{S}$} & \multicolumn{1}{c}{$\mathrm{T}$} & $\mathrm{T}-\mathrm{AG}$ & $\mathrm{ET}$ & ET-AG \\
\hline $\mathrm{pH}$ & $7.36 \pm 0.03$ & $7.33 \pm 0.03$ & $7.30 \pm 0.04^{*}$ & $7.30 \pm 0.02^{*}$ & $7.26 \pm 0.01^{*}$ \\
$\mathrm{BE}(\mathrm{mmol} / \mathrm{L})$ & $-1.28 \pm 1.10$ & $-2.78 \pm 0.55$ & $-3.28 \pm 1.20$ & $-2.88 \pm 2.10$ & $-4.17 \pm 1.40^{*}$ \\
$\mathrm{HCO}_{3}^{-}(\mathrm{mmol} / \mathrm{L})$ & $23.82 \pm 1.23$ & $23.72 \pm 1.28$ & $23.64 \pm 0.96$ & $24.60 \pm 1.51$ & $24.98 \pm 1.74$ \\
$\mathrm{pCO}_{2}(\mathrm{mmHg})$ & $41.18 \pm 3.47$ & $45.02 \pm 2.48$ & $45.12 \pm 3.07$ & $50.33 \pm 2.7^{\star *}$ & $49.92 \pm 2.32^{*}$ \\
$\mathrm{pO}_{2}(\mathrm{mmHg})$ & $58.99 \pm 5.37$ & $53.81 \pm 2.34$ & $56.10 \pm 5.45$ & $55.03 \pm 4.00$ & $56.78 \pm 5.82$ \\
$\mathrm{SatO}_{2}(\%)$ & $75.30 \pm 2.91$ & $67.97 \pm 2.98$ & $72.03 \pm 4.77$ & $65.74 \pm 4.84^{*}$ & $64.96 \pm 7.27^{*}$ \\
\hline
\end{tabular}

S: sedentary; T: trained; T-AG: trained and supplemented with alanyl-glutamine; ET: trained and subjected to an exhaustive test; ET-AG: trained group supplemented with alanyl-glutamine and subjected to an exhaustive test; $\mathrm{BE}$ : base excess; $\mathrm{HCO}_{3}{ }^{-}$: bicarbonate; $\mathrm{pCO}_{2}$ : carbon dioxide partial pressure; $\mathrm{pO}_{2}$ : oxygen partial pressure: SatO $\mathrm{O}_{2}$ : oxygen saturation. Data are reported as means $\pm \mathrm{SD}$. ${ }^{*} \mathrm{P}<0.05$ compared to $\mathrm{S}$ group; ${ }^{\#} \mathrm{P}<0.05$ compared to T group (ANOVA followed by Bonferroni post hoc test).

\section{Gasometric parameters}

The gasometric data are presented in Table 1. Compared to the $S$ group, animals from the T-AG, ET, and ET$A G$ groups had lower $\mathrm{pH}(\mathrm{P}<0.05)$. The animals of the ET-AG group had lower BE compared to the $S$ group $(P<0.05)$. Only the animals subjected to the exhaustive test had significantly higher $\mathrm{pCO}_{2}$ compared to the $\mathrm{S}$ group. In addition, animals from the ET group also had higher $\mathrm{pCO}_{2}$ than animals of the $\mathrm{T}$ group $(\mathrm{P}<0.05)$. Animals from the ET and ET-AG groups had lower $(\mathrm{P}<0.05) \mathrm{SatO}_{2}$ compared to sedentary animals.

\section{Impact of AG supplementation on intestinal permeability}

Figure 2A shows that animals of the ET group increased $(P<0.05)$ lactulose excretion compared to the $\mathrm{S}, \mathrm{T}$, and T-AG groups $(32.83 \pm 4.12$ vs $7.34 \pm 2.05,10.59 \pm$ 3.75 , and $11.03 \pm 3.76 \%$, respectively). AG treatment attenuated $(P<0.05)$ this phenomenon in the ET-AG group $(32.83 \pm 4.12$ vs $19.73 \pm 5.14 \%)$. We also observed an increase of the mannitol urinary excretion for the T-AG animals compared to sedentary animals (Figure 2B). The ET group showed higher $(P<0.05)$ mannitol levels $(45.49 \pm$ $5.12 \%)$ than the $\mathrm{S}, \mathrm{T}$, and T-AG groups (13.91 \pm 2.91 , $22.33 \pm 4.06$, and $26.18 \pm 4.78 \%$, respectively). In addition, ET-AG animals presented mannitol excretion levels of $45.07 \pm 7.75$ showing that $A G$ supplementation did not significantly alter mannitol excretion. Figure $2 \mathrm{C}$ shows that the lactulose/mannitol excretion ratio was higher $(P<0.05)$ in the ET group compared to the $S$ group $(0.76 \pm 0.12$ vs $0.49 \pm 0.11 \%)$. It was also higher $(P<0.05)$ in the ET group compared to the ET-AG $(0.76 \pm 0.12$ vs $0.52 \pm 0.05 \%)$.

\section{Gene transcription of cellular intestinal transporters and tight junction}

Analyzing oligopeptide transporter 1 (PepT-1) transcription, T and ET groups exhibited a significant upregulation $(1.03 \pm 0.27$ vs $4.99 \pm 2.1, P=0.016$ and $3.87 \pm 1.35$, $\mathrm{P}=0.016$, respectively) compared to $\mathrm{S}$ animals (Figure $3 \mathrm{~A}$ ).
In addition, T-AG and ET-AG showed no statistical differences compared to the sedentary animals $(2.59 \pm 2.08$ and $1.64 \pm 1.29$, respectively). The same behavior is seen in Figures $4 \mathrm{~A}$ and $5 \mathrm{~A}$, in which there were no differences comparing T vs T-AG (1.08 \pm 0.45 and $0.56 \pm 0.45$, respectively) or ET vs ET-AG $(0.92 \pm 0.25$ and $0.44 \pm 0.35$, respectively).

For gene expression analysis of the tight junction zona ocludens-1 (ZO-1), Figure $3 \mathrm{~B}$ shows upregulation in the animals from the $\mathrm{T}$ and ET groups when these groups were compared to the $S$ group $(9.44 \pm 3.52, P=0.016$ and $6.76 \pm 2.15, P=0.029$ vs $1.71 \pm 1.21$ ), respectively. When we compared T-AG and ET-AG (Figure $3 \mathrm{~B}$ ) to the S group, there were no significant differences $(3.63 \pm 3.33$ and $3.38 \pm 2.55$ vs $1.71 \pm 1.21$, respectively). To determine if AG was completely reverting ZO-1 expression, we compared the group T vs T-AG (Figure 4B) and ET vs ET-AG (Figure 5B). However, we noticed that there was no statistical difference among these two comparisons (1.05 \pm 0.39 vs $0.41 \pm 0.37$ and $0.79 \pm 0.25$ vs $0.48 \pm 0.25$, respectively).

After analyzing the ileum gene expression of occludin (Figure $3 C$ ), we observed that when group $S$ was compared to all others (T, T-AG, ET, and ET-AG) only animals from group $\mathrm{T}$ were significantly upregulated $(1.25 \pm 0.99$ vs $4.87 \pm 1.74, \mathrm{P}=0.029 ; 1.30 \pm 1.53 ; 2.51 \pm 0.52 ; 0.88 \pm$ 0.37 , respectively). These data showed that $A G$ reverted the expression of occludin in the T-AG group. To verify if this reversion was complete, we compared group $T$ with T-AG (Figure 4C). Our data showed that $T$ animals were also statistically different from T-AG animals, indicating that there was a complete reversion of occludin expression after AG supplementation (1.38 \pm 0.49 vs $0.13 \pm 0.14$, $\mathrm{P}=0.03)$. In addition, occludin expression of group ET was statistically different from group ET-AG (0.96 \pm 0.20 vs $0.36 \pm 0.15, P=0.03$; Figure $5 C$ ).

As shown in Figure 3D, when the sedentary group was compared to all other groups (T, T-AG, ET, and ET-AG), claudin-2 transcription was upregulated only for the $E T$ 

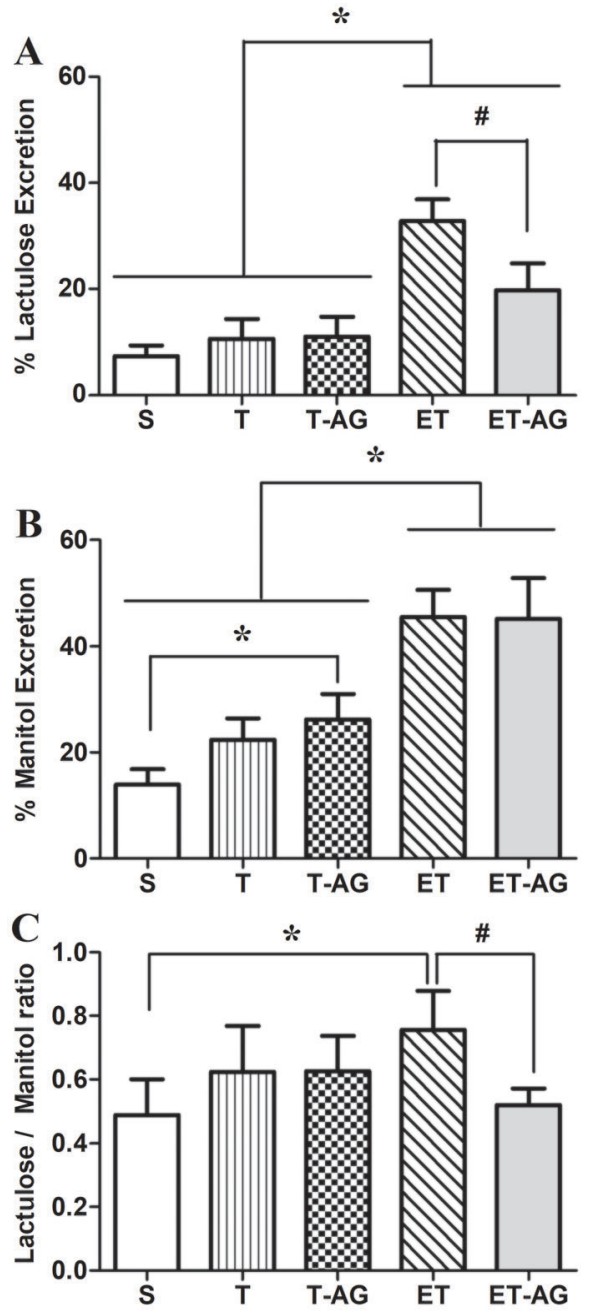

Figure 2. Intestinal permeability was evaluated by the percentage of lactulose, mannitol, and lactulose/mannitol ratio. Animals were subdivided into 5 groups: sedentary without supplementation (S), trained animals without supplementation $(T)$, trained animals supplemented with alanyl-glutamine (T-AG), trained animals subjected to an exhaustive test without supplementation (ET), and trained animals subjected to an exhaustive test and supplemented with alanyl-glutamine (ET-AG). The data are reported as means $\pm S D(n=8)$. ${ }^{*} P<0.05$ compared with $S$; ${ }^{\#} P<0.05$ compared with ET (ANOVA followed by Bonferroni post hoc test).

group $(1.12 \pm 0.53$ vs $0.50 \pm 0.25,0.83 \pm 0.31,3.46 \pm 1$, $P=0.03$, and $1.58 \pm 0.88$, respectively). To verify if $A G$ supplementation was really reverting the mRNA expression of claudin-2 (Figure 5D), we performed the comparison of ET vs ET-AG. A statistical difference was found between ET and ET-AG (1.26 \pm 0.45 vs $0.44 \pm 0.18$, $\mathrm{P}=0.03)$. However, we could not see any statistical difference between groups $T$ and T-AG for claudin-2 mRNA expression $(0.77 \pm 0.39$ vs $1.27 \pm 0.47)$.

\section{Discussion}

The present study showed that 12 weeks of periodized training substantially enhanced the performance of rats subjected to an exhaustive test protocol. After a period of physical training, the rats subjected to long-term exhaustive exercise showed increased paracellular intestinal permeability, which was attenuated by $A G$ pretreatment. In addition, AG mediated other effects in animals that were not submitted to the exhaustive test.

The impact of physical exercise on the gastrointestinal tract is well known. In this sense, we recently reported that acute exercise delays the gastric emptying rate in rats, a phenomenon prevented by bicarbonate treatment. In addition, extracellular acidosis in vitro inhibits cholinergic neurotransmission in the rat gastric fundus by selectively influencing the $\mathrm{Gq} / 11$ protein-signaling pathway $(22,23)$. Now, we report for the first time the morphofunctional modifications in the intestinal barrier, such as increased intestinal permeability associated with changes in the transcription of a tight junction (claudin-2) in a physically exhaustive test, which was prevented by AG treatment.

The impact of exercise on gut permeability was assessed by the non-invasive lactulose/mannitol assay, which is considered a gold standard method (24). The intestinal transport of mannitol and lactulose are directly related to transcellular and paracellular permeability, respectively. Thus, damage to epithelial cells and tight junctions are associated with decreased mannitol and increased lactulose absorption (25). In the present work, both lactulose and mannitol excretion increased in trained rats subjected to exhaustive exercise. In pre-clinical and clinical studies, high urinary excretion of both probes is indicative of the leaky gut syndrome $(26,27)$. It is suggested that during maximum exercise, the blood is shunted away from the viscera, and splanchnic blood flow can decrease by as much as $80 \%$ (28). Therefore, strenuous exercise can impair the gastrointestinal blood flow, making the gut mucosa susceptible to ischemic injury and increasing the intestinal permeability. Physical exercise at a $70 \% \mathrm{VO}_{2}$-max intensity was also recently shown to increase the intestinal permeability in humans (29). In the present study, the trained animals subjected to the exhaustive test swam for almost $4 \mathrm{~h}$, and the stress and lack of splanchnic nutrition could have contributed to increase intestinal permeability. Our finding reinforces a recent study, where runners demonstrated increase intestinal permeability, regardless of the presence of gastrointestinal symptoms (30). In addition, both groups subjected to exhaustive exercise showed signs of physical stress related to acidosis and hypercapnia. Thus, we suggest that the duration of this stress for a longer period of time in trained animals could explain their increase in gut permeability.

The tight junction proteins have important functions for intestinal epithelial tissue, and several pathophysiological 

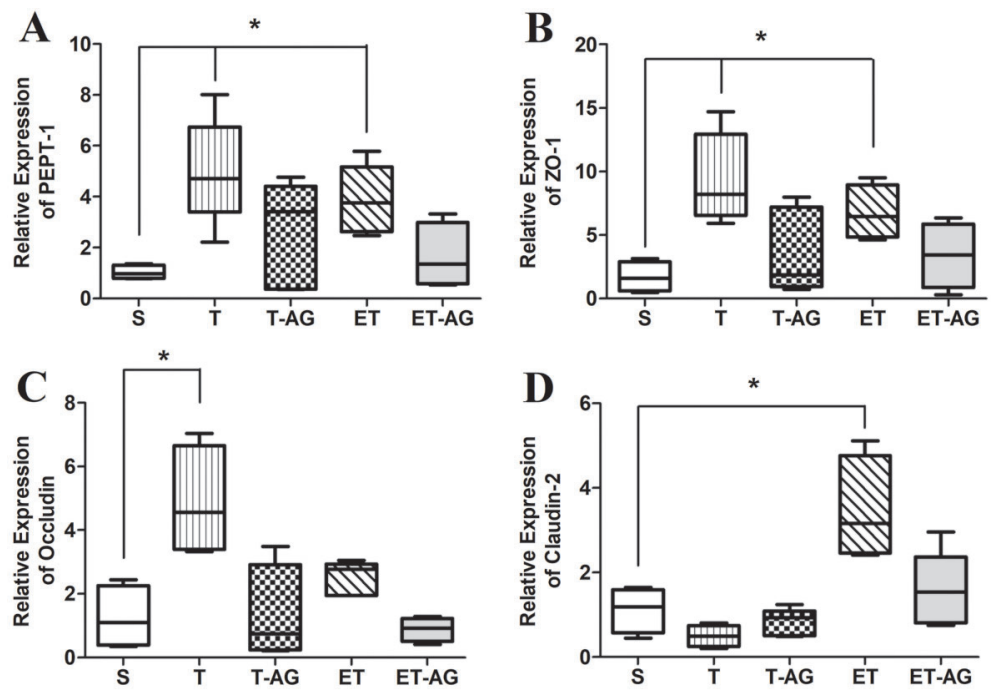

Figure 3. Relative mRNA expression of (A) peptide transporter-1 (PEPT-1), (B) zonula occludens-1 (ZO-1), (C) occludin, and (D) claudin-2. Animals were subdivided into 5 groups: sedentary without supplementation (S), trained animals without supplementation (T), trained animals supplemented with alanyl-glutamine (T-AG), trained animals subjected to an exhaustive test without supplementation $(E T)$, and trained animals subjected to an exhaustive test and supplemented with alanyl-glutamine (ET-AG). The data are reported as median, minimum and maximum, and interquartile range $(n=8)$. ${ }^{*} P<0.05$ compared with the $S$ group (Mann-Whitney test).
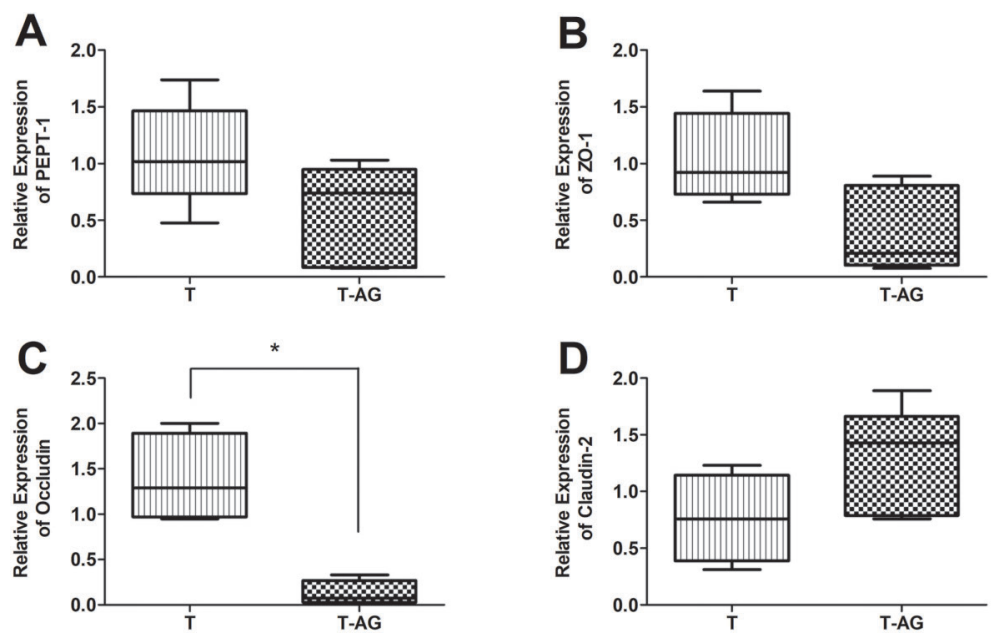

Figure 4. Relative mRNA expression of (A) peptide transporter-1 (PEPT-1), (B) zonula occludens-1 (ZO-1), (C) occludin, and (D) claudin-2 between trained animals without supplementation (T group) and trained animals supplemented with alanyl-glutamine (T-AG). The data are reported as median, minimum and maximum, and interquartile range $(n=8)$. ${ }^{*} P<0.05$ compared with the $T$ group (Mann-Whitney test).

conditions modify its morphofunctional configuration (31). In the present study, ZO-1 and occludin mRNA up-regulation could be related to morphological adaptations promoted by physical exercise, but not associated with increasing gut permeability. In the present study, training induced occludin and ZO-1 mRNA up-regulation. This may explain why these animals did not exhibit alterations in gut permeability. The overexpression of these mRNAs may indicate higher resistance of the tight junction assembly (29). ZO-1 serves as an intermediary in the contact between occludin and the actin cytoskeleton, and the upregulation of ZO-1 and occludin mRNA would confirm the hypothesis of higher tight junction resistance. These morphological adaptations promoted by physical exercise were somehow regulated by $A G$ supplementation. Further studies are necessary to clarify the exact molecular 

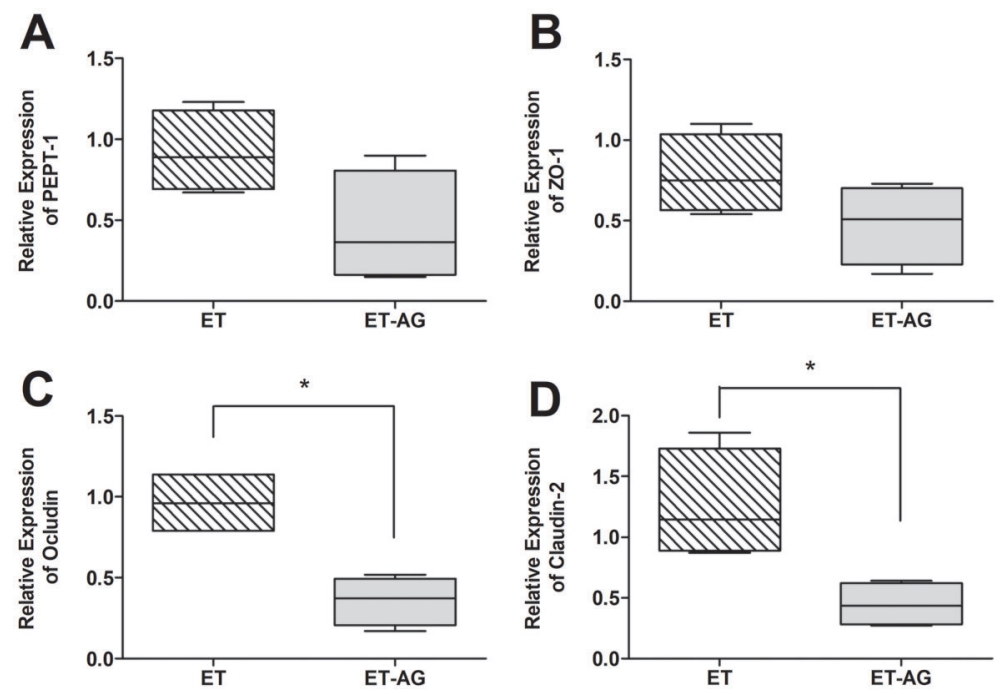

Figure 5. Relative mRNA expression of (A) peptide transporter-1 (PEPT-1), (B) zonula occludens-1 (ZO-1), (C) occludin, and (D) claudin-2 between trained animals submitted to an exhaustive test without supplementation (ET group) and trained animals submitted to an exhaustive test supplemented with alanyl-glutamine (ET-AG). The data are reported as median, minimum and maximum, and interquartile range $(n=8)$. ${ }^{*} P<0.05$ compared with the ET group (Mann-Whitney test).

mechanism in which AG interfered on tight junction mRNA expression.

However, training did not interfere with claudin-2 expression. Claudin-2 knockout mice showed small intestine paracellular damage with malabsorption of nutrients and electrolytes (32). Claudin proteins are considered primary seal-forming proteins, and have the ability to polymerize into linear fibrils, (33). Loss of claudin-2 expression has been shown to compromise paracellular intestinal absorption of essential nutrients resulting in premature death of mice (32). We suggest that claudin-2 mRNA upregulation possibly contributed to the increased intestinal permeability observed in the present study. In addition, increased protein expression of claudin-2 is associated with lower transepithelial electrical resistance and higher cation selectivity (34). Recently, intestinal leakage has been correlated with increased gene transcription of claudin-2 and critical modifications of the intestinal microbiota (35). Moreover, the increased intestinal permeability in runners is strongly linked to microbiome modifications during prolonged exercise (36).

One goal in the present study was to determine whether chronic AG treatment would be beneficial to intestinal permeability after a swim challenge. Glutamine is the main fuel used by intestinal mucosa cells and other rapidly proliferating tissues (37). Because of its superior physicochemical solubility and favorable thermal stability in acid medium (38), glutamine was replaced by dipeptide AG. It is well established that glutamine absorption is enhanced after the ingestion of AG compared to the free amino acids (39). This dipeptide is transported into enterocytes by PepT-1. We found that PepT-1 mRNA was upregulated in the groups that underwent training ( $T$ and ET groups). It is conceivable that physical activity requires adaptations for more nutrients to be absorbed, and an increase in PepT-1 mRNA expression would be one of such adaptations. However, our data suggested that AG supplementation returned the PepT-1 mRNA expression to physiological condition, since the groups that received $A G$ were not different from the sedentary group. Our analysis revealed no differences in PepT-1 mRNA expression between the $T$ and $T-A G$ groups or between the ET and ET-AG groups. Such phenomenon should be investigated further, but intestinal tissue appeared to be more responsive to PepT-1 mRNA expression during exercise since this tissue may need more energetic subtracts provided by $A G$.

AG treatment did not change the rats' swimming performance; none of the biochemical or gasometrical indices differed in AG-supplemented groups. However, biomarkers of paracellular route were decreased by $A G$ treatment. AG also protected claudin-2 mRNA overexpression. These findings reinforced that $A G$ had modulating mechanisms on the structural proteins present in tight junctions. Some studies showed that glutamine deprivation decreases transepithelial resistance of tight junction proteins, including claudin-1 and occludin (40). Although Noth and coworkers (11) reported that glutamine improved intestinal permeability dysfunction by increasing occludin expression, we did not observe occludin mRNA upregulation in the groups that were subjected to the exhaustive test (11). 
In conclusion, chronic AG administration protected intestinal barrier function against the impact of acute exhaustive exercise by reducing intestinal permeability and modulated the mRNA expression of claudin-2. These findings emphasize the importance of $A G$ during physical exercise for gut physiology and support its possible use among endurance athletes.

\section{Supplementary Material}

Click here to view [pdf].

\section{References}

1. Martin D. Physical activity benefits and risks on the gastrointestinal system. South Med J 2011; 104: 831-837, doi: 10. 1097/smj.0b013e318236c263.

2. ter Steege RW, Van der Palen J, Kolkman JJ. Prevalence of gastrointestinal complaints in runners competing in longdistance run: an internet-based observational study in 1281 subjects. Scan J Gastroenterol 2008; 43: 1477-1482, doi: 10.1080/00365520802321170.

3. Horner KM, Schubert MM, Desbrow B, Byrne NM, King NA. Acute exercise and gastric emptying: a meta-analysis and implications for appetite control. Sports Med 2015; 45: 659678, doi: 10.1007/s40279-014-0285-4.

4. Silva MT, Palheta-Junior RC, Sousa DF, Fonseca-Magalhães PA, Okoba W, Campos CP, et al. Sodium bicarbonate treatment prevents gastric emptying delay caused by acute exercise in awake rats. J Appl Physiol 2014; 116: 11331141, doi: 10.1152/japplphysiol.01242.2013.

5. de Oliveira EP, Burini RC, Jeukendrup A. Gastrointestinal complaints during exercise: prevalence, etiology, and nutritional recommendations. Sports Med 2014; 44: S79-S85, doi: 10.1007/s40279-014-0153-2.

6. Lambert GP. Role of gastrointestinal permeability in exertional heatstroke. Exerc Sport Sci Rev 2004; 32: 185-190, doi: 10.1097/00003677-200410000-00011.

7. Tota L, Piotrowska A, Palka T, Morawska M, Mikul'áková W, Mucha D, et al. Muscle and intestinal damage in trithletes. PLos One 2019; 14: e0210651, doi: 10.1371/journal.pone. 0210651.

8. van Wijck K, Lenaerts K, Grootjans J, Wijnands KA, Poeze $M$, Van Loon LJ, et al. Physiology and pathophysiology of splanchnic hypoperfusion and intestinal injury during exercise: strategies for evaluation and prevention. Am J Physiol Gastrointest Liver Physiol 2012; 303: G155-G168, doi: 10.1152/ajpgi.00066.2012.

9. Costa RJS, Camões-Costa V, Snipe RMJ, Dixon D, Russo I, Huschtscha Z. The impact of exercise induced hypohydration on gastrointestinal integrity, function, symptoms, and systemic endotoxin and inflammatory profile. J Appl Physiol 2019; 126: 1281-1291, doi: 10.1152/japplphysiol.01032.2018.

10. Rodrigues RS, Oliveira RA, Li Y, Zaja-Milatovic S, Costa LB, Braga-Neto $M B$, et al. Intestinal epithelial restitution after TcdB challenge and recovery from Clostridium difficile infection in mice with alanyl-glutamine treatment. $J$ Infect Dis 2013; 207: 1505-1515, doi: 10.1093/infdis/jit041.

\section{Acknowledgments}

This work was supported by grants from Coordenação de Aperfeiçoamento de Pessoal de Nível Superior (CAPES), Conselho Nacional de Pesquisas (CNPq), and Fundação Cearense de Apoio ao Desenvolvimento Científico e Tecnológico (FUNCAP). This study was part of the M.Sc. dissertation in Pharmacology presented by A.K.L. Freitas to the School of Medicine, Federal University of Ceará. The authors thank Mr. Haroldo Pinheiro for his skillful technical assistance.

11. Noth R, Häsler R, Stüber E, Ellrichmann $M$, Schäfer $H$, Geismann $\mathrm{C}$, et al. Oral glutamine supplementation improves intestinal permeability dysfunction in a murine acute graft-vs-host disease model. Am J Plysiol Gastrointest Liver Physiol 2013; 304: G646-G654, doi: 10.1152/ajpgi.00246. 2012.

12. Lima NL, Soares AM, Mota RM, Monteiro HS, Guerrant RL, Lima AA. Wasting and intestinal barrier function children taking alanil-glutamine-supplement enteral formula. J Pediatr Gastroenterol Nutr 2007; 44: 365-374, doi: 10.1097/MPG.0b 013e31802eecdd.

13. Rogero MM, Tirapegui J, Pedrosa RG, Castro IA, Pires ISO. Effect of alanyl-glutamine supplementation on plasma and tissue glutamine concentrations in rats submitted to exhaustive exercise. Nutrition 2006; 22: 564-571, doi: 10.1016/ j.nut.2005.11.002.

14. Tezini GC, Silveira LC, Villa-Clé PG, Jacinto CP, Di Sacco $\mathrm{TH}$, Souza HC. The effect of aerobic physical training on cardiac autonomic control of rats submitted to ovariectomy. Menopause 2009; 16: 110-116, doi: 10.1097/gme.0b013e3 $18182 \mathrm{~d} 352$.

15. Sant'Ana JE, Pereira MG, Dias da Silva VJ, Dambrós C, Costa-Neto CM, Souza HC. Effect of the duration of daily aerobic physical training on cardiac autonomic adaptations. Autonom Neurosci 2011; 159: 32-37, doi: 10.1016/j.autneu. 2010.07.006.

16. Beck WR, Botezelli JD, Pauli JR, Ropelle ER, Gobatto CA. Melatonin has an ergogenic effect but does not prevent inflammation and damage in exhaustive exercise. Sci Rep 2015; 16: 5:18065, doi: 10.1038/srep18065.

17. Zhang $\mathrm{H}$, Liu M, Zhang $\mathrm{Y}$, Li X. Trimetazidine attenuates exhaustive exercise-induced myocardial injury in rats via regulation of the Nrf2/NF-kB signaling pathway. Front Pharmacol 2019; 10: 175, doi: 10.3389/fphar.2019.00175.

18. Carneiro-Filho BA, Lima IP, Araújo DH, Cavalcanti MC, Carvalho $\mathrm{GH}$, Brito $\mathrm{GA}$, et al. Intestinal barrier function and secretion in methotrexate-induced rat intestinal mucositis. Dig Dis Sci 2004; 49: 65-72, doi: 10.1023/B:DDAS.00000 11604.45531.2c.

19. Lima AA, Silva TM, Gifoni AM, Barrett LJ, McAuliffe IT, Bao Y. Mucosal injury and disruption of intestinal barrier function in HIV-infected individuals with and without diarrhea and cryptosporidiosis in northeast Brazil. Am J Gastroenterol 1997; 92: 1861-1866, PMID: 9382053. 
20. Li B, Matter EK, Hoppert HT, Grayson BE, Seeley RJ, Sandoval DA. Identification of optimal reference genes for RT-qPCR in the rat hypothalamus and intestine for the study of obesity. Int J Obes 2014; 38: 192-197, doi: 10.1038/ ijo.2013.86.

21. Livak KJ, Schmittgen TD. Analysis of relative gene expression data using real-time quantitative PCR and the 2(-Delta Delta C(T)) Method. Methods 2001; 25: 402-408, doi: 10.1006/meth.2001.1262.

22. de Oliveira DMN, Batista-Lima FJ, De Carvalho EF, Havt A, da Silva MTB, Dos Santos AA, et al. Extracellular acidosis selectively inhibits pharmacomechanical coupling induced by carbachol in strips of rat gastric fundus. Exp Physiol 2017;102: 1607-1618, doi: 10.1113/EP086573.

23. Cavalcante $A K M$, Siqueira RCL, Feitosa Júnior VN, de Andrade CR, Santos AA, da Silva MTB. Acute exercise inhibits gastric emptying of liquids in rats: influence of the NO-cGMP pathway. Braz J Med Biol Res 2018; 51: e7541, doi: 10.1590/1414-431x20187541.

24. Kosek M, Guerrant RL, Kang G, Bhutta Z, Yori PP, Gratz J, et al. Assessment of environmental enteropathy in the MALED cohort study: theoretical and analytic framework. Clin Infect Dis 2014; 59: S239-S247, doi: 10.1093/cid/ciu457.

25. Gervasoni J, Primiano A, Graziani C, Scaldaferri F, Gasbarrini A, Urbani A, et al. Validation of UPLC-MS/MS Method for Determination of Urinary Lactulose/Mannitol. Molecules 2018; 23. pii: E2705, doi: 10.3390/molecules23102705.

26. Odenwald MA, Turner JR. Intestinal permeability defects: is it time to treat? Clin Gastroenterol Hepatol 2013; 11: 1075-1083, doi: 10.1016/j.cgh.2013.07.001.

27. Nguyen TD, Prykhodko $O$, Hållenius FF, Nyman $M$. Monobutyrin reduces liver cholesterol and improves intestinal barrier function in rats fed high-fat diets. Nutrients 2019; 11. pii: E308, doi: 10.3390/nu11020308.

28. ter Steege RW, Kolkman JJ. The pathophysiology and management of gastrointestinal symptoms during physical, and the role of splanchnic blood flow. Aliment Pharmacol Ther 2012; 35: 516-528, doi: 10.1111/j.1365-2036.2011.04980.x.

29. Zuhl MN, Lanphere KR, Kravitz L, Mermier CM, Schneider $\mathrm{S}$, Dokladny K, et al. Effects of oral glutamine supplementation on exercise-induced gastrointestinal permeability and tight junction protein expression. J Appl Physiol 2014; 116: 183-191, doi: 10.1152/japplphysiol.00646.2013.

30. Karhu E, Forsgård RA, Alanko L, Alfthan H, Pussinen $P$, Esa Hämäläinen $E$, et al. Exercise and gastrointestinal symptoms: running-induced changes in intestinal permeability and markers of gastrointestinal function in asymptomatic and symptomatic runners. Eur J Appl Physiol 2017; 117: 2519-2526, doi: 10.1007/s00421-017-3739-1.
31. Thomas YM, Prashant N, Rana Al-Sadi. Tight Junctions and the Intestinal Barrier. Physiology of the Gastrointestinal Tract In: Elsevier Inc.; 2018. p. 587-639.

32. Wada M, Tamura A, Takahashi N, Tsukita S. Loss of claudins 2 and 15 from mice causes defects in paracellular $\mathrm{Na}^{+}$flow and nutrient transport in gut and leads to death from malnutrition. Gastroenterology 2013; 144: 369-380, doi: 10.1053/j.gastro.2012.10.035.

33. Furuse $M$, Hata $M$, Furuse $K$, Yoshida $Y$, Haratake $A$, Sugitani $Y$, et al. Claudin-based tight junctions are crucial for the mammalian epidermal barrier: a lesson from claudin1-deficient mice. J Cell Biol 2002; 156: 1099-1111, doi: 10. 1083/jcb.200110122.

34. Furuse M, Furuse K, Sasaki H, Tsukita S. Conversion of zonulae occludentes from tight to leaky strand type by introducing claudin-2 into Madin-Darby canine kidney I cells. J Cell Biol 2001; 153: 263-272, doi: 10.1083/jcb.153. 2.263 .

35. Brown EM, Wlodarska M, Willing BP, Vonaesch $\mathrm{P}$, Han J, Reynolds LA, Arrieta MC. et al. Diet and specific microbial exposure trigger features of environmental enteropathy in a novel murine model. Nat Commun 2015; 6: 7806, doi: 10.1038/ncomms8806.

36. Karl JP, Margolis LM, Madslien EH, Murphy NE, Castellani JW, Gundersen Y, et al. Changes in intestinal microbiota composition and metabolism coincide with increased intestinal permeability in young adults under prolonged physiological stress. Am J Physiol Gastrointest Liver Physiol 2017; 312: G559-G571, doi: 10.1152/ajpgi.00066.2017.

37. Zuhl M, Dokladny K, Mermier C, Schneider S, Salgado R, Moseley P. The effects of acute oral glutamine supplementation on exercise-induced gastrointestinal permeability and heat shock protein expression in peripheral blood mononuclear cells. Cell Stress Chaperones 2015; 20: 85-93, doi: 10.1007/s12192-014-0528-1.

38. Leite RD, Lima NL, Leite CA, Farhat CK, Guerrant RL, Lima AA. Improvement of intestinal permeability with alanylglutamine in HIV patients: a randomized, double blinded, placebo-controlled clinical trial. Arq Gastroenterol 2013; 50: 56-63, doi: 10.1590/S0004-28032013000100011.

39. Harris RC, Hoffman JR, Allsopp A, Routledge NB. Lglutamine absorption is enhanced after ingestion of Lalanylglutamine compared with the free amino acid or wheat protein. Nutr Res 2012; 32: 272-277, doi: 10.1016/j.nutres. 2012.02.003.

40. Li N, Lewis P, Samuelson D, Liboni K, Neu J. Glutamine regulates Caco-2 cell tight junction proteins. Am J Physiol Gastrointest Liver Physiol 2004; 287: G726-G733, doi: 10. 1152/ajpgi.00012.2004. 